\title{
Abdominal Pain in an Elderly Patient
}

\section{Case}

An 89-year-old woman presented to the ED with the chief complaints of abdominal pain and nausea with vomiting. The patient stated that several hours prior, she had ingested an expired beverage, which she related to the sudden onset of her symptoms. The patient denied fever, chills, dysuria, or frequency. Her medical history was significant for chronic atrial fibrillation (AF) and congestive heart failure. The patient's medications included metoprolol and furosemide; she was not on any anticoagulation medication.

On physical examination, the patient appeared her stated age, and was in moderate distress secondary to the abdominal pain. Vital signs were: temperature, $98.8^{\circ} \mathrm{F}$; heart rate, 98 beats/ min; respiratory rate, 20 breaths/min; and blood pressure, 116/72 mm Hg. Oxygen saturation was $97 \%$ on room air. The head, eyes, ears, nose, and throat examination was unremarkable. On lung examination, breath sounds were equal bilaterally with bibasilar rales. The heart rhythm was irregularly irregular without murmurs, rubs, or gallops. The abdomen was soft to palpitation, but diffusely tender, without rebound, guarding, or mass. Rectal examination revealed normal tone and brown stool, and was trace positive for heme.

The emergency physician (EP) ordered an electrocardiogram (ECG), complete blood count, basic metabolic profile (BMP), urinalysis, and lipase test. The patient was administered intravenous (IV) normal saline at $75 \mathrm{cc} / \mathrm{h}$, and morphine 4 $\mathrm{mg}$ and ondansetron $4 \mathrm{mg}$ IV for the abdominal pain, nausea, and vomiting. She required several more doses of morphine due to the severity of the pain. The laboratory results included an elevated white blood count of $18.4 \times 10^{9} / \mathrm{L}$ with a left shift, but normal hemoglobin and hematocrit values. The ECG demonstrated $\mathrm{AF}$ with a controlled ventricular rate; there was no evidence of ischemia or injury. The BMP was remarkable for a slightly depressed potassium level (3.3 $\mathrm{mEq} / \mathrm{L}$ ), a decreased serum bicarbonate of $20 \mathrm{mEq} / \mathrm{L}$, and evidence of renal insufficiency with a blood urea nitrogen of $28 \mathrm{mg} / \mathrm{dL}$ and a serum creatinine of $1.6 \mathrm{mg} /$ dL. Given the ongoing severe pain, leukocytosis, metabolic acidosis, and lack of clear etiology, the EP ordered a computed tomography (CT) scan of the abdomen and pelvis; no IV contrast was ordered because of the abnormal renal function studies.

The radiologist interpreted the CT scan as essentially normal. The EP admitted the patient to the on-call hospitalist, who consulted both cardiology and gastroenterology services. During the night, the patient com-

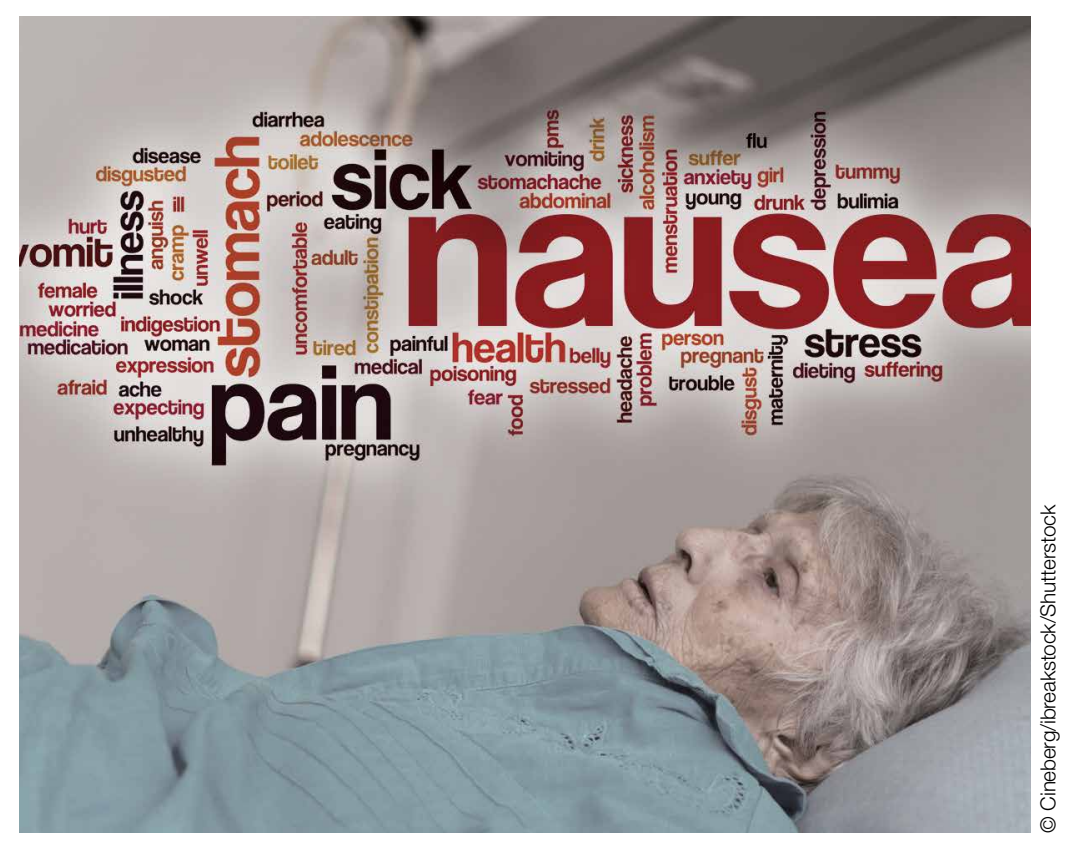

plained of increasing abdominal pain, and her abdomen became distended with peritoneal signs. She was taken emergently to the operating room in the early morning hours. A large segment of gangrenous small intestine was found upon exploration. The surgery was discontinued and comfort care measures were instituted. The patient died the following day. 
The patient's family sued the EP and the hospital for failure to make a timely diagnosis of mesenteric ischemia. They further stated that the EP should have ordered a CT angiogram (CTA) of the abdomen and pelvis. The defense argued that a contrast CT scan was contraindicated because of the patient's poor renal function. A defense verdict was returned at trial.

\section{Discussion}

Elderly patients (defined as older than age 65 years) presenting to the ED with abdominal pain remain a diagnostic challenge for even the most seasoned clinician. While elderly patients with a chief complaint of abdominal pain represent only a small percentage of ED patients, approximately $50 \%$ to $66 \%$ of these patients will require hospitalization, while one-third will require a surgical intervention. ${ }^{1}$ The seriousness of this complaint in elderly patients is further emphasized by the fact that older patients with abdominal pain have a 6- to 8-fold increase in mortality compared to younger patients. ${ }^{2,3}$ This can be partially explained by the simple fact that the life-threatening causes of abdominal pain-abdominal aortic aneurysm, mesenteric ischemia, bowel perforation, volvulus, and acute bowel obstruction-occur more frequently (but not exclusively) in elderly patients. Historical risk factors for life-threatening causes of abdominal pain include: age older than 65 years, immunocompromised state, alcohol abuse, cardiovascular (CV) disease (eg, coronary artery disease, hypertension, AF), major comorbidities (eg, cancer, renal failure), and prior surgery or recent gastrointestinal instrumentation. ${ }^{1}$

The patient in this case had two risk factors for lifethreatening causes of lower abdominal pain-age and AF. These are also two of the major risk factors for mesenteric ischemia, which was her ultimate diagnosis.

Acute mesenteric ischemia refers to the sudden onset of small intestinal hypoperfusion, frequently due to acute occlusion (embolism or thrombosis) of an intestinal artery, most commonly the superior mesenteric artery (SMA). ${ }^{4}$ The SMA supplies the entire small intestine except for the proximal duodenum. Other causes of acute mesenteric ischemia include venous occlusion (thrombosis) and nonocclusive mesenteric ischemia secondary to vasoconstriction from low-cardiac output or use of vasopressors. ${ }^{4}$

Thromboembolic occlusion of the SMA is the most common cause of acute mesenteric ischemia, accounting for $67 \%$ to $95 \%$ of cases. ${ }^{4}$ In addition to $\mathrm{AF}$, the risk of arterial embolism is increased in patients with valvular disease, infective endocarditis, recent myocardial infarction, aortic atherosclerosis, or aortic aneurysm. ${ }^{4}$ Risk factors for thrombotic arterial occlusion include peripheral artery disease, advanced age, and low-cardiac output states. ${ }^{5}$

A frequent presentation of embolic mesenteric arterial ischemia, occurring in approximately one-third of cases, is an elderly patient with AF (or other source of embolism) and onset of severe, sudden abdominal pain out of proportion to physical examination. While nausea and vomiting are also common, bloody bowel movements are less frequent in the early course of the disease process. ${ }^{4} \mathrm{~A}$ history of a prior embolic event is present in approximately one-third of such patients.

On physical examination, the abdomen may be normal initially, or demonstrate only mild distention and tenderness without peritoneal signs. However, as the ischemia progresses, the abdomen becomes more distended, bowel sounds become absent, and peritoneal signs (ie, guarding and rebound) become apparent. ${ }^{6}$

The results of laboratory studies can suggest the diagnosis, but none are confirmatory. Laboratory findings may include a marked leukocytosis with left shift, an elevated hematocrit secondary to hemoconcentration, and metabolic acidosis. A helpful clinical pearl is to consider intestinal ischemia in the differential diagnosis of any patient with acute abdominal pain and metabolic acidosis. ${ }^{6}$ Serum lactate is frequently elevated (73\%-94\%) but a very nonspecific marker. Similarly, an arterial blood gas analysis may demonstrate metabolic acidosis. More recently, a normal D-dimer result has been used to help exclude the diagnosis of acute intestinal ischemia, since it is elevated in $96 \%$ of patients with the disease. ${ }^{6}$ Similar to lactate, an abnormal D-dimer result has a poor specificity $(40 \%) .{ }^{6}$ Early in the disease course, nearly all laboratory studies may be normal.

Depending on the severity of the presentation, imaging can help make the definitive diagnosis. For patients with peritonitis or obvious bowel perforation, IV fluid resuscitation, IV antibiotics, and immediate surgical exploration are indicated. Plain radiographs of the abdomen offer little help, as many of the findings early in the disease course are nonspecific, and radiographs can be normal in $25 \%$ of cases. ${ }^{6}$ Ultrasound can identify arterial stenosis or occlusion of the SMA, but is frequently technically limited by the presence of air-filled loops of distended bowel. ${ }^{6}$ Magnetic resonance angiography has 
similar sensitivity and specificity as CTA for mesenteric arterial ischemia, and is actually more sensitive than CTA for mesenteric venous thrombosis; it also can be performed in patients with contrast allergy. ${ }^{6}$ However, CTA is performed more commonly because of its lower cost, greater speed, and wide availability. ${ }^{6}$ A CTA of the abdomen and pelvis (without oral contrast) is probably the best study for patients in whom mesenteric ischemia is high on the differential diagnosis. ${ }^{6}$ For patients with a less clear picture and a broader differential diagnosis, a CT scan of the abdomen/pelvis with both IV and oral contrast is preferred. ${ }^{7}$ Common findings on CT scan with IV/oral contrast in acute mesenteric ischemia include the following: bowel wall thickening, dilatation, stranding, bowel wall attenuation, abnormal enhancement, and pneumatosis. Unfortunately, many of these findings are nonspecific. ${ }^{7}$

Once the diagnosis of acute mesenteric ischemia is made, patients should be designated "nothing by mouth" and a nasogastric tube placed to decompress the bowel. These patients will require IV fluid resuscitation with normal saline. The amount and rate will depend on their clinical presentation and underlying CV status. Any electrolyte abnormalities should be corrected and broad spectrum IV antibiotics initiated. Vascular surgery or general surgery services should be consulted to determine the optimal management. Most patients with acute intestinal ischemia due to mesenteric arterial occlusion (or venous occlusive or nonocclusive mesenteric ischemia) will be started on anticoagulation, typically IV heparin, unless contraindications are present. ${ }^{6}$ Surgical treatment options include arterial embolectomy, arterial bypass, arterial stenting, arterial thrombolysis, or intra-arterial vasodilator infusion. ${ }^{6}$

\section{References}

1. Kendall JL, Moreira ME. Evaluation of the adult with abdominal pain in the emergency department. UpToDate Web site. http://www. uptodate.com/contents/evaluation-of-the-adult-with-abdominal-painin-the-emergency-department. Updated September 29, 2016. Accessed November 30, 2016.

2. Lewis LM, Banet GA, Blanda M, Hustey FM, Meldon SW, Gerson LW. Etiology and clinical course of abdominal pain in senior patients: a prospective, multicenter study. J Gerontol A Biol Sci Med Sci. 2005;60(8):1071-1076.

3. Sanson TG, O'Keefe KP. Evaluation of abdominal pain in the elderly. Emerg Med Clin North Am. 1996;14(3):615.

4. Tendler DA, Lamont JT, Pearl G. Acute mesenteric arterial occlusion. UpToDate Web site. http://www.uptodate.com/contents/acute-mesenteric-arterial-occlusion. Updated May 27, 2015. Accessed November 30, 2016.

5. McKinsey JF, Gewertz BL. Acute mesenteric ischemia. Surg Clin North Am. 1997;77(2):307-318.

6. Tendler DA, Lamont JT. Overview of intestinal ischemia in adults. UpToDate Web site. http://www.uptodate.com/contents/overview-ofintestinal-ischemia-in-adults. Updated February 23, 2016. Accessed November 30, 2016.

7. Wiesner W. Khurana B, Ji H, Ros PR. CT of acute bowel ischemia. Radiology. 2003;226(3):635-650. 